\title{
Automatic Phase Detection for Stochastic On-Chip Traffic Generation
}

\author{
Antoine Scherrer ${ }^{*}$ \\ LIP - ENS Lyon \\ 46 , allée d'Italie \\ 69364, Lyon cedex 7, France \\ ascherre@ens-lyon.fr
}

\author{
Antoine Fraboulet, Tanguy Risset \\ CITI - INSA Lyon \\ 21, avenue Jean Capelle \\ 69621 Villeurbanne Cedex, France \\ firstname.lastname@insa-lyon.fr
}

\begin{abstract}
During System on Chip (SoC) design, Network on Chip (NoC) prototyping is used for adapting NoC parameters to the application running on the chip. This prototyping is currently done using traffic generators which emulate the SoC components (IPs) behavior: processors, hardware accelerators, etc. Traffic generated by processor-like IPs is highly non-regular, it must be decomposed into program phases. We propose an original feature for NoC prototyping, inspired by techniques used in processor architecture performance evaluation: the automatic detection of traffic phases. Integrated in our $\mathrm{NoC}$ prototyping environment, this feature permits to have a completely automatic toolchain for the generation of stochastic traffic generators. We show that our traffic generators emulate precisely the behavior of processors and that our environment is a versatile tool for networks-on-chip prototyping. Simulations are performed in a SystemC-based simulation environment with a mesh network-on-chip (DSPIN) and a processor running MP3 decoding applications.
\end{abstract}

Categories and Subject Descriptors: C.4 [Computer Systems Organization] : Performance of Systems - Modeling techniques.

General Terms: Algorithms, Performance, Experimentation.

Keywords: Traffic generation, Network-on-chip, Phase behavior, Stochastic modeling, Performance evaluation.

\section{INTRODUCTION}

Systems on chip (SoC) are now commonly used in embedded systems for multimedia and telecommunication applications. Most of these SoC are composed of a single processor controlling various components (Intellectual Property: IP) all connected together. The computing power required by

${ }^{*}$ This work has been partly founded by CNRS and STMicroelectronics

Permission to make digital or hard copies of all or part of this work for personal or classroom use is granted without fee provided that copies are not made or distributed for profit or commercial advantage and that copies bear this notice and the full citation on the first page. To copy otherwise, to republish, to post on servers or to redistribute to lists, requires prior specific permission and/or a fee.

CODES+ISSS'06, October 22-25, 2006, Seoul, Korea.

Copyright 2006 ACM 1-59593-370-0/06/0010 ...\$5.00. emerging applications running on mobile terminals, such as video on mobile phone for instance, has induced the development of a more complex SoC infrastructure, the so-called multi-processor SoC (MPSoC) typically composed of a number of master components (processors or DMA for hardware accelerators) connected to a network-on-chip (NoC) or a hierarchy of busses.

The advent of networks-on-chip (NoC) has significantly increased the design complexity of such systems with some hard problems related to parallelism such as memory and cache coherency, non-determinism, efficient workload distribution, and network contention. Solving these problems during the short time available for design requires fundamental improvements in design methodologies. The most important shift is the setting of a refinement methodology allowing designers to explore design space at various levels of precision. These levels, called transaction, bus-accurate, synthesizable, allow the designer to check quickly that performances related to various metrics are achieved before writing the complete description of the system.

During design space exploration, simulation time is a major problem. There are two run-time behaviors very difficult to model at a high level: cache behavior and network contention. Precise simulation of these two behaviors can only be done with a low-level description of the components. This means hours (sometimes days) of simulation for a single execution or, as it is usually preferred, the use of extremely expensive hardware emulators. Reducing simulation time can be achieved by a clever analysis of the behavior of the system during execution. We are interested in the simulation of onchip network behavior and performance evaluation. Traffic generators (TG) are more and more used during SoC design for platform prototyping or performance evaluation. When using TGs, simulation time is decreased because the IP is not fully simulated. Simulation is also more flexible.

Most recent traffic generation methods use stochastic models. Statistical analysis and synthesis of on-chip traffic is difficult because this traffic usually presents complex statistical behavior. As pointed out by [13], the behavior of application code is decomposed into phases which have very different characteristics. Each phase can appear several times during the complete execution of the program. The precision of the simulation and the possibility of integrating the whole process in an automatic (or at least semi-automatic) framework are important parameters for evaluating the usefulness of a traffic generation environment.

In this paper, we present the automatic detection of traffic phases by analyzing simulation traces and show that 
these phases are necessary to emulate the traffic generated by multi-media applications running on SoC. By gathering different features presented individually in various recent works, our traffic generation environment provides a very flexible tool for networks-on-chip prototyping. It can run a deterministic traffic replay (as in [9]) or generate a stochastic traffic with first order (as in [6]) and second order (as in [15]) statistics adjusted to a particular trace. The feature described in this paper is the ability to run a traffic divided into separate phases, each phase having different characteristics. This makes our TG able to capture the inherent non-stationarity present in the traffic generated by the processors. We validate the precision of our traffic simulation in SystemC. We show that the network latency, transaction delay and aggregated throughput of a complete SoC platform are very close when we use TGs replacing processors. With our environment, the designer can explore the design space in a very flexible manner by, for instance, exploring other network architectures on a single phase in which network contention occurs.

The paper is organized as follows. In Section 2 we review the different existing techniques of traffic generation. Section 3 presents our traffic generator and the flow that we propose for analyzing and synthesizing on-chip traffic. Section 4 presents our experimental results highlighting the points mentioned above.

\section{RELATED WORK}

Using traffic generators in a simulation platform involves the following steps: $i$ ) the IP designer collects simulation traces by observing the behavior at the interface of each master component (if the IP is available), ii) he builds traffic models as close as possible to these traces, iii) the platform designer instantiates a traffic generator for each master component based on these models, and $i v$ ) inserts them in the simulation platform in place of the original components. Traffic generators can be separated into two main categories: the deterministic approach, in which traffic is produced using a finite state machine (FSM) configured by the IP designer or using a previous simulation trace, and the stochastic approach, in which the traffic is produced by a parameterized non-deterministic process.

Deterministic traffic generators (TG) $[3,9,7]$ are derived from real simulation traces or written from scratch by IP designers. Such TGs can generate accurate transactions in time, size, and idle time that match the behavior of an IP. The advantages of this approach are the precision and the speedup factor it can achieve compared to the complete IP simulation. However, one limitation of the deterministic approach is that the length of the simulation is limited by the length of input traces used. Furthermore, such TGs cannot handle behaviors that are dependent on input data sets.

An alternative solution is to use stochastic traffic generators. These TGs build a model of the traffic. Such a modeling permits to study how small variations in the model parameters impact performance. This is an interesting way of testing NoC robustness with reasonably accurate traffic. Such a model can also be useful when the IP is not fully available or when the behavior is likely to change slightly from one execution to the other. However some traffics are very difficult to model and the traffic generation environment should include advanced statistical analysis tools such as multi-phase statistical analysis and second order statis- tics fit. For instance Marculescu et al. [15] have isolated a long-range-dependent behavior (i.e. second order statistical properties) at the coarse-grain level. Our simulation environment is currently able to capture and generate traffic with second order statistic adjusted to a particular covariance [12]. However, our experiments do not exhibit longrange dependence (even in the execution of the MPEG2 application not reported here). In the work of Marculescu [15], the MPEG2 is executed by hardware accelerators, and longrange-dependence has been observed in the communications between the accelerators, whereas we use a software implementation of the MPEG2 algorithm running on a processor and its associated cache. This result has to be confirmed by other simulations; our conjecture is that the absence of long-range dependence is due to the presence of caches that smooth the communications.

The major part of NoC performance evaluation is currently done using random sources $[16,14,6]$. These works mainly focus on the evaluation of the NoC in its early stage of development, and on its performance under random traffic. However none of these works propose a fitting procedure to determine the adequate statistical models that should be used to simulate the traffic: most of them choose arbitrarily the statistical behavior of each IP. Moreover to our knowledge, none of these approaches have introduced multi-phase modeling. A complete traffic generation environment should integrate both deterministic and stochastic traffic generation techniques.

A processor associated with a cache generates a non-stationary traffic, which can be divided into phases corresponding to different parts of the executed program. Each phase is stationary in the sense that its stochastic characteristics are almost constant. This point has been thoroughly investigated in the domain of processor architecture performance evaluation, a very good summary of which is presented by Calder et al. in [13]. Calder et al. isolate program phases by analyzing basic blocs repartition in successive intervals (an interval can represent 10 millions of instructions). Then, these phases are compared and grouped using a $k$-means algorithm [8]. We have adopted a similar approach to decompose the traffic generated by a processor in phases. Our model is simpler and the interval is approximately composed of a thousand of transactions. The data used to represent the activity of the processor is the traffic's statistical characteristics.

Calder et al. use such a phase decomposition in SimPoint [5] for architecture performance evaluation. This is a powerful technique that can provide huge improvements in simulation by simulating only one simulation point per phase and replicating the behavior during all the corresponding phases. We do not pursue the same goal here because we target precise traffic simulation of a given IP for NoC prototyping. Network contention needs to be precisely simulated, and as it is the result of the superposition of several traffics, picking simulation points becomes a difficult task. However further studies should be done, based for instance on the work of [2] to see if the use of simulation points may be applicable for NoC prototyping.

\section{MPTG ENVIRONMENT}

We now present our analysis and synthesis flow for building multi-phase traffic generators that can be used to replace an IP in cycle-accurate NoC performance evaluation. 


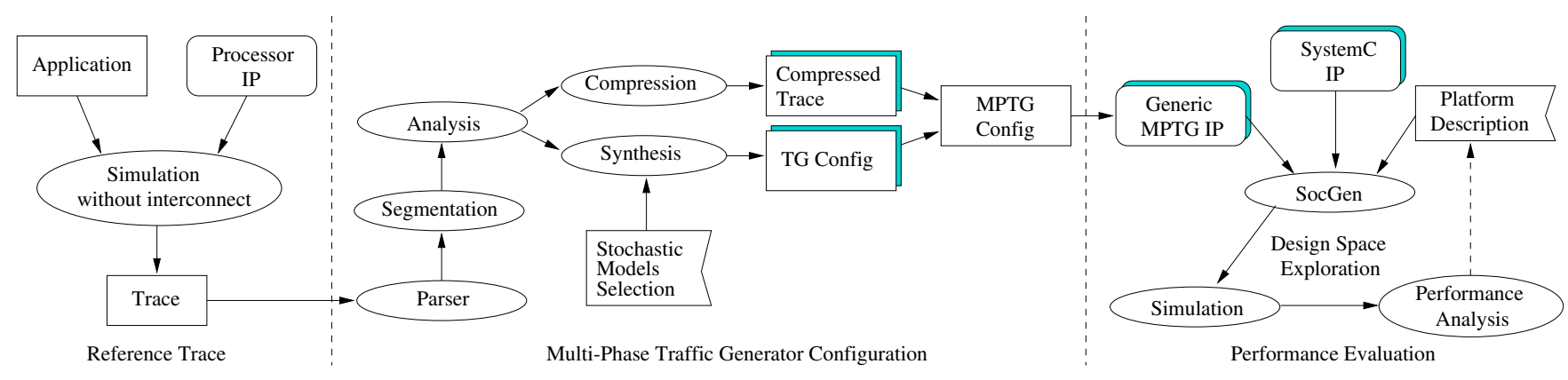

Figure 1: MPTG Framework: Traffic analysis and synthesis flow

\subsection{On-chip traffic modelling}

The traffic produced by a component is modelled as a sequence of transactions. The $i^{\text {th }}$ transaction is a 4 -uple $(A(i), C(i), S(i), D(i))$ meaning in this order, target address, command (read or write), size of transaction, and delay (number of cycles between two successive requests). This is illustrated in Figure 2. From the transaction sequence, we define the aggregated throughput $W_{\Delta}(j)$, which corresponds to the amount of bus-words transferred in the time interval $[j \Delta,(j+1) \Delta]$. We also define the latency of the $i^{t h}$ transaction $L(i)$ as the number of cycles between the start of a $i^{\text {th }}$ request and the start of the associated response. It basically corresponds to the round-trip time in the network.

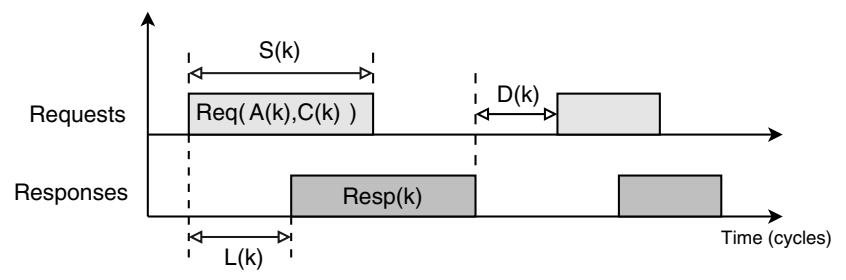

Figure 2: Traffic modelling formalism

Traffic is generated according to the 4-uple describing each transaction, and this 4-uple can be either read from a previously recorded trace (replay), or generated as the realization of a stochastic process.

\subsection{Global methodology}

The global simulation flow is depicted on Figure 1. First, we generate a reference trace by simulating the processor IP to be emulated. This trace is obtained with an ideal network environment (no network contention), which makes the simulation very fast. Then, we process the trace in our traffic analysis and synthesis tool and we obtain configuration files for the traffic generators. A parametric generic traffic generator has been written once for all, it is referred to further in the text as MPTG. Transactions are generated by MPTG according to a phase description file and a sequencer is in charge of switching between phases. Each phase consists either of a replay of a recorded trace, or of a stochastic model with parameters adjusted by the fitting procedure described in [12]. Finally, the platform designer describes the desired platform architecture (such as the one presented in Figure 3) and uses a perl script (referred to as SocGen) that generates all files needed for simulation. Thus, the simulation takes place and performance analysis indicates whether some parameters of the platform have to be changed or not.

Two important features of our MPTG are the following: $i$ ) it is aware of the network latency (requests are sent only if the network is ready), and $i i$ ) it can be configured to emulate the communication scheme of the target IP. For example, as we target processor/cache traffic, the MPTG is configured with blocking reads and non-blocking writes in order to emulate the write buffer of the cache. These properties ensure that the same MPTG configuration files can be used on various on-chip interconnects, thus allowing fast design space exploration of the NoC.

\subsection{Automatic phase determination}

The contribution of the paper lies in the adaptation of the work of [13] to NoC prototyping. In general, decomposing a non-stationary process into stationary parts is very difficult. Nevertheless, it appears that our programs are piece-wise stationary. We use the $k$-means algorithm [8] which is a classical technique to group multi-dimensional values in similar sets, and we end up with a good clustering as demonstrated in section 4 . The worst case complexity of this algorithm is exponential but it is in practice very fast. Our automatic phase determination algorithm is the following:

1. First, we select a list of $M$ elements of the transaction sequence (delay, size, command, address, see Section 3.1).

2. The transaction sequence is then split into non-overlapping intervals of length $L$ transactions. Mean and variance are computed on each interval and for each selected element, so we build a $2 M$-dimensional representative vector used for the clustering.

3. We perform clustering in $k$ phases using the $k$-means algorithm with different values of $k$ (2 to 7 in practice). The algorithm finds $k$ centroids in the space of representative vectors. Each interval will be assigned the number of its closest center (in the sense of the quadratic distance).

4. To evaluate different clustering, we compute the Bayesian Information Criterion (BiC) [10]. The $\mathrm{BiC}$ gives a score of the clustering and a higher $\mathrm{BiC}$ means better clustering.

Once the phases are identified, statistical analysis is performed on each extracted phase by an automatic fitting procedure that adjusts the first and second statistical orders 

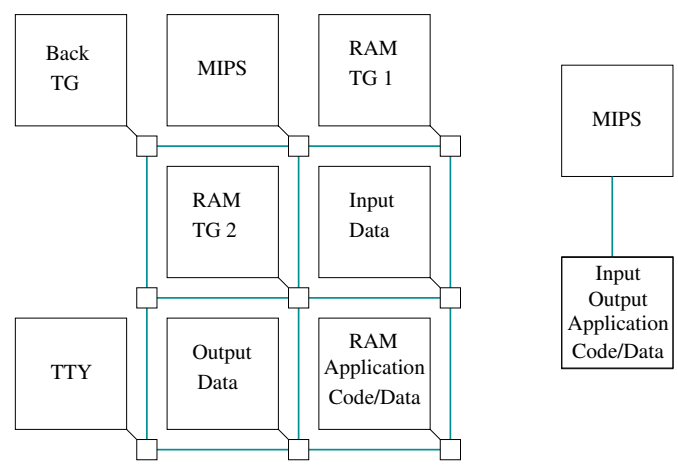

Figure 3: Simulation platforms: direct (right) and mesh1 (left)

(see [12]) for details). The designer has to choose which model he wants to use before analyzing the trace. We developed an independent random number generator that can produce realizations of a wide variety of processes [12]. The latter generator is integrated in the MPTG and the analysis produces the adequate MPTG configuration file.

\section{EXPERIMENTATIONS}

Hereafter, we present experimental results. With these results, we want to demonstrate that $i$ ) the automatic segmentation of traffic traces is efficient, and $i i$ ) the accuracy of stochastic multi-phase traffic generation is good. The speedup factor of MPTG is of the same order as in [9] (2$5 \mathrm{x})$, and more experiments are presented in [11].

\subsection{Experimental setup}

We use an open source, SystemC-based, cycle-accurate and bit-accurate simulation environment: SocLib [1]. We use a tiny operating system for multiprocessor management (Mutek). We present here the results on an implementation of the MPEG-layer 3 audio decoding software, further referred to as MP3. 2 frames are decoded in the results presented here, representing 350000 memory transactions when executed on the processor (MIPS r3000). Similar results have been obtained for MPEG2 and JPEG 2000 applications, but are not presented here because of space limitation. We use the DSPIN NoC, inherited from the research of the LIP6 laboratory (evolution of SPIN [4]). It uses wormhole memorization strategy and $X Y$ routing. The processor caches includes both data and instructions. It is composed of 32 lines of 8 words. Aggregated throughput (further simply referred to as throughput) has been computed as the number of flits transferred in consecutive time window of size 100 cycles.

We denote by platform a particular physical interconnection of various IPs. We used two platforms:

- The direct platform does not use any interconnect, the processor is directly connected to a memory holding all necessary data. The latency is thus constantly equal to 1 cycle. This platform is used for basic validation of the MPTG and for reference trace collection as shown in Figure 1.

- The mesh1 platform is shown in Figure 3. The components are interconnected with the DSPIN NoC. The MIPS processor is running the application. The Back TG is used for introducing contention over the network. It sends requests to both memories RAM TG1 and RAM TG2, whereas

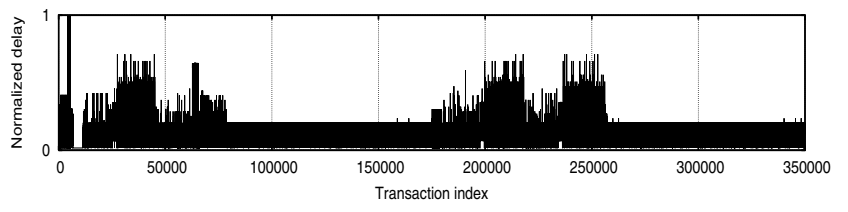

(a) original trace

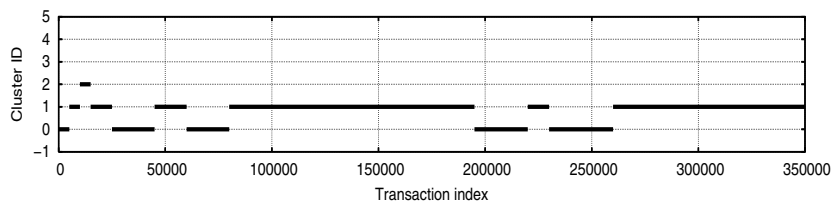

(b) 3-means clustering

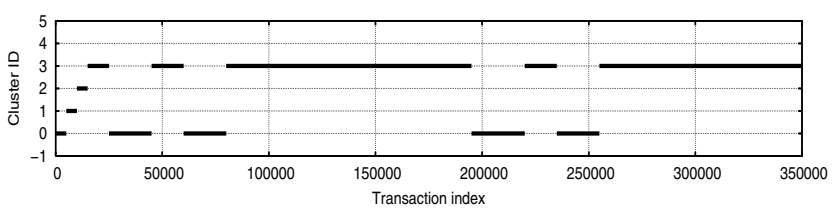

(c) 4-means clustering

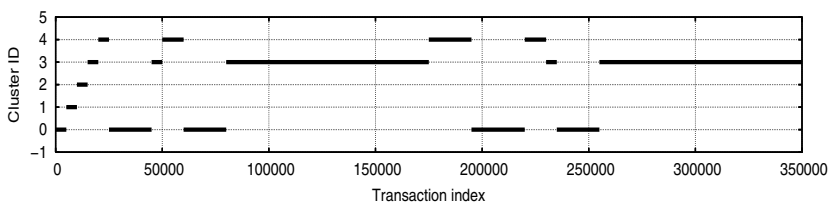

(d) 5-means clustering

Figure 4: Phases discovered by our algorithm on the MP3 traffic trace using the delay, for different phase numbers.

the MIPS communicates with the three other memories used for code, data, input and output streams. In order to test the MPTG in a more realistic way, the Back TG alternates between two phases, one with a high communication load and another with a low one. This introduces a time-varying contention and approximately multiplies the number of cycles of the execution by 3 .

\subsection{Segmentation of the MP3 application}

Figure 4a shows the delays of transactions $D(i)$ as a function of the transaction index $i$. One can distinguish the boot at the beginning, and then the two frames being decoded. For each frame, several phases can be identified (for instance a long one at the end) and two important points have to be highlighted. Firstly, the time evolution of the traffic is not stationary, so a stochastic fit on the whole trace would be meaningless. Secondly, similar behaviors appear, hence a segmentation should be done. This was already observed in the high performance computing community [13], however it is, to our knowledge, the first time a traffic trace is being analyzed in this way.

We have run the phase segmentation algorithm described in Section 3.3 for different values of $k$, using the delay element. The size of intervals was set to 5000 transactions. The choice of $k$ is a trade-off between statistical accuracy (we need large interval for statistical estimators to converge) and phase grain (we need many intervals in order to well identify the traffic phases). The chosen value is such a good trade-off on the analyzed trace. Figures $4 \mathrm{~b}, 4 \mathrm{c}$ and $4 \mathrm{~d}$ show the results for various number of phases. One can see that 


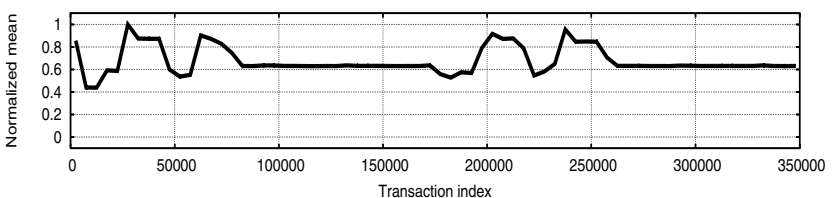

(a) Mean delay evolution

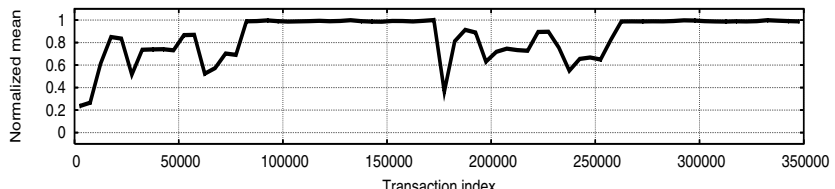

(b) Mean size evolution

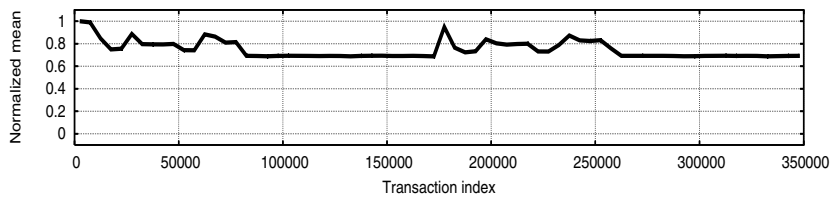

(c) Mean command evolution

Figure 5: Evolution of the mean of different elements (delay, size, command) of the MP3 application, computed in intervals of size $L=5000$ transactions.

the algorithm finds the analogy between the two frames, and identifies phases inside each one of them. The segmentation seems valid and pertinent. As the segmentation is done with mean and variance as representative vectors, one expects that each identified phase exhibits a stationary behavior, likely to be processed by a stochastic analysis.

\begin{tabular}{|c|c|c|c|c|c|c|c|}
\hline & 2 & 3 & 4 & 5 & 6 & 7 & 8 \\
\hline Delay & 85.3 & 76.1 & 76.9 & $\mathbf{1 2 6}$ & 123 & 109 & 100 \\
\hline Size & 6.6 & 44.5 & $\mathbf{7 3 . 8}$ & 44.3 & 34.1 & 28.2 & 16.7 \\
\hline Command & -91 & -53 & $\mathbf{- 2 3}$ & -53 & -63 & -69 & -80 \\
\hline
\end{tabular}

Table 1: Bayesian Information Criterion (BiC) for each element (rows), and various numbers of phases (columns). Highest value per row is highlighted.

As explained in Section 3.3, we have computed Bayesian Information Criterions (BiC) of the clustering done using different elements (delay, size and command) and different numbers of phases. Address sequence is not considered in these results because the dynamics of address values is such that the clustering fails. Table 1 shows the $\mathrm{BiC}$ values, each row corresponds to an element, and each column to a number of phases. For the size and command elements, the highest $\mathrm{BiC}$ value (best clustering) is achieved for $k=4$, and for the delay element it is $k=5$. As the $\mathrm{BiC}$ is always higher for a clustering using the delay element, we further only use this element.

\subsection{Accuracy of the traffic generation}

Let us first detail the statistical analysis introduced in Section 3.2. In order to build MPTG configuration files, we automatically compute for each identified phase: the probability distribution function of the delay, the access probability of each memory segment, and for each segment, the read/write probability. As we are emulating a processor and its cache, we fixed the read transaction size to the cache line size, and we also computed the probability distribution function of the write transaction's sizes.

We have performed simulations with different configurations: "MIPS", which is the reference simulation of the MP3 application running on the MIPS, "MPTG-N" for which traffic is generated with a MPTG with $N$ phases, "DR" which corresponds to a deterministic replay (the trace has been recorded and is replayed), and "RANDOM" which is a constant rate traffic with uniformly distributed target selection (the rate is fixed to the mean observed rate). Each configuration is run on both DIRECT and MESH1 platforms.

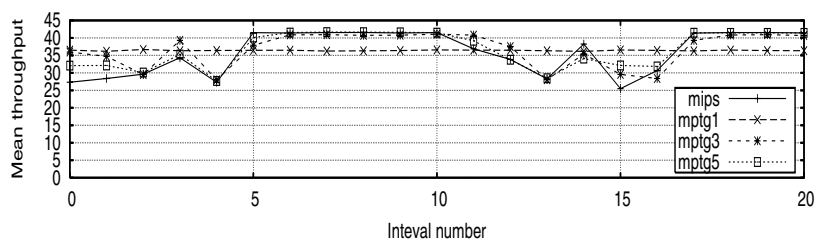

Figure 6: Evolution of the mean throughput of the MP3 application on the direct platform. Each interval is 100000 cycles long.

In order to compare a given configuration with the MIPS reference one, we should not look at global metrics such as the average delay or the average throughput. This would not highlight the interest of the multi-phase approach. So, we have defined an accuracy measure that can be computed on each element (delay, size, command and throughput). We compare the mean evolution of the metrics, just as represented in figure 5, for both simulations (MIPS and the one under study). This can be done graphically as depicted in figures 6 and 7. But, to summarize the results we defined the error as the mean of absolute values of relative differences between two mean evolutions. Let $M_{\text {ref }}(i)$ be the mean evolution of some element for the reference simulation, let further $M(i)$ be the evolution of the same element for another simulation, and let finally $n$ be the number of points of both functions. The error (in percent) reads: $E r r=\frac{1}{n} \sum_{i}\left|M_{r e f}(i)-M(i)\right| / M_{\text {ref }}(i) * 100$ and is reported in tables 2 and 3. As expected, the higher the phase number is, the more accurate the results are. This underlines the importance of multi-phase traffic generation. Accuracy is lower on the MESH1 platform because the stochastic nature of traffic generation has a stronger impact. Still the multi-phase stochastic traffic generation lies in between the very accurate deterministic replay and the very inaccurate random traffic.

The cycle error (cycle column is tables 2 and 3 ) is computed as the relative difference between numbers of simulated cycles and therefore concerns the whole simulation. The various MPTG configurations (including the one with one phase only) all have a low error on that metric, whereas the RANDOM configuration exhibits a non-negligible one. This result shows that MPTG provides a good emulation of the average traffic, the delay error is thus compensated on the whole simulation. Despite of the fact that the average rate was used in the construction of the RANDOM the configuration, the number of simulated cycles is far from the one of the reference simulation. This emphasize the need for advanced statistical analysis for on-chip traffic generation. 


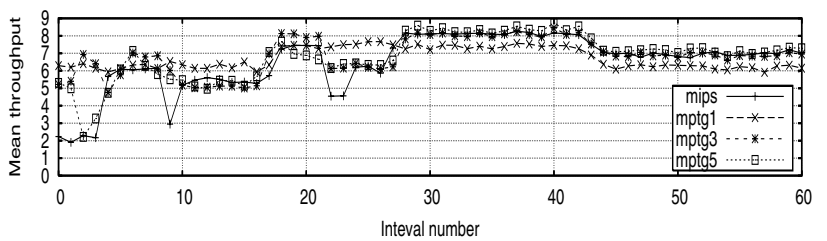

Figure 7: Evolution of the mean throughput of the MP3 application on the mesh1 platform. Each interval is 100000 cycles long.

\begin{tabular}{|c|c|c|c|c|c|c|}
\hline Config. & Delay & Size & Cmd & Thput & Latency & Cycle \\
\hline DR & 0 & 0 & 0 & 0 & 0 & \\
\hline RANDOM & 31.78 & 34.31 & 6.91 & 13.64 & 0 & 32.1 \\
\hline MPTG1 & 7.83 & 15.18 & 6.43 & 11.45 & 0 & 1.83 \\
\hline MPTG3 & 1.97 & 8.34 & 3.28 & 5.58 & 0 & 1.55 \\
\hline MPTG5 & 1.33 & 3.27 & 1.15 & 2.60 & 0 & 1.21 \\
\hline
\end{tabular}

Table 2: Error (in percent) on various metrics with respect to the MIPS reference simulation (direct platform).

Figures 6 and 7 show the evolution of the throughput of the different configurations on respectively the DIRECT and on the MESH1 platforms. The MPTG1 is a straight line (one phase only) on DIRECT. On the MESH1 platform, the sort of wave oscillation in Figure 7 is a consequence of the two traffic phases of the Back TG introducing a time-varying contention on the NoC. One can see that MPTG3 and MPTG5 configurations follow, as expected, the evolution of the reference simulation.

\begin{tabular}{|c|c|c|c|c|c|c|}
\hline Config. & Delay & Size & Cmd & Thput & Latency & Cycle \\
\hline DR & 1.15 & 0 & 0 & 0.20 & 0.12 & 1.7 \\
\hline RANDOM & 41.28 & 75.24 & 7.71 & 102.32 & 27.83 & 11.3 \\
\hline MPTG1 & 18.60 & 14.76 & 6.26 & 12.7 & 10 & 2.83 \\
\hline MPTG3 & 17.19 & 8.17 & 3.26 & 6.21 & 0.78 & 2.81 \\
\hline MPTG5 & 14.77 & 3.24 & 1.21 & 5.65 & 0.63 & 2.75 \\
\hline
\end{tabular}

Table 3: Error (in percent) on various metrics with respect to the MIPS reference simulation (mesh1 platform).

These results show that multi-phase stochastic traffic generation is worth a try for NoC prototyping. Even though it is not as precise as deterministic replay, the phase behavior of the IP is preserved, which is in our opinion a key point for emulating the true contention on the network. The choice between stochastic and deterministic traffic generation depends on the purpose of the study. For instance random traffic generation is a good way to evaluate and compare routing strategies and other large scale design choices, whereas deterministic trace replay can provide a good accuracy for tuning the implementation details in the routers. We believe that the multi-phase stochastic traffic generation is interesting as a compromise between random and deterministic approaches. It combines a reasonable accuracy and overcomes deterministic limitations. It especially provides the designer a phase description of traffic, and a stochastic model for each identified phase. This allows more flexibility in the traffic generation. For instance, the parameters of the models can be slightly changed in order to evaluate the robustness of the NoC.

\section{CONCLUSION AND FUTURE WORKS}

In this paper, we have explained how traffic phases are automatically identified and synthesized in our traffic generation environment. Experimental results show that this automatic clustering is meaningful and that it can be performed on various elements of the transaction sequence. Such a feature, coupled with the advanced stochastic analysis, fitting and synthesis procedure already available, makes our traffic generation environment an efficient NoC prototyping tool. Experimental results show the accuracy and the versatility of our MPTG, highlighting some of its key features : accurate replay over various interconnections, multi-phase traffic generation, stochastic traffic analysis and generation. Future works include the study of other applications, and the investigation of simulation time reduction using the phase behavior of each IP of the SoC.

\section{REFERENCES}

[1] Soclib simulation environment. On-line, available at http://soclib.lip6.fr/, 2005.

[2] M. V. Biesbrouck, T. Sherwood, and B. Calder. A co-phase matrix to guide simultaneous multithreading simulation. In SPASS, 2004.

[3] N. Genko, D. Atienza, G. D. Micheli, J. M. Mendias, R. Hermida, and F. Catthoor. A complete network-on-chip emulation framework. In DATE, pages 246-251, 2005.

[4] A. Greiner and P. Guerrier. A generic architecture for on-chip paquets-switched interconnections. In DATE, 2000.

[5] G. Hamerly, E. Perelman, and B. Calder. How to use simpoint to pick simulation points. Sigmetrics Perform. Eval. Rev., 31(4):25-30, 2004.

[6] K. Lahiri, S. Dey, and A. Raghunathan. Evaluation of the traffic-performance characteristics of system-on-chip communication architectures. In $V L S I D$ '01, pages 29-35, 2001.

[7] M. Loghi, F. Angiolini, D. Bertozzi, L. Benini, and R. Zafalon. Analyzing on-chip communication in a MPSoC environment. In DATE, pages 752-757, 2004.

[8] J. MacQueen. Some methods for classification and analysis of multivariate observations. In Berkeley Symposium on Mathematical Statistics and Probability, pages 281-297, 1967.

[9] S. Mahadevan and et al. A network traffic generator model for fast network-on-chip simulation. In DATE, pages 780-785, 2005 .

[10] D. Pelleg and A. Moore. X-means: Extending k-means with efficient estimation of the number of clusters. In International Conference on Machine Learning, pages 727-734, San Francisco, 2000.

[11] A. Scherrer, A. Fraboulet, and T. Risset. A generic multi-phase on-chip traffic generation environment. In $A S A P, 2006$. to appear.

[12] A. Scherrer, N. Larrieu, P. Owezarski, P. Borgnat, and P. Abry. Non gaussian and long memory statistical characterisations for internet traffic with anomalies. Technical report, LIP - ENS Lyon, 2005.

[13] T. Sherwood, E. Perelman, G. Hamerly, S. Sair, and B. Calder. Discovering and exploiting program phases. IEEE Micro, 23(6):84-93, 2003.

[14] R. Thid, M. Millberg, and A. Jantsch. Evaluating NoC communication backbones with simulation. In 21th IEEE Norchip Conference, Riga, Nov. 2003.

[15] G. Varatkar and R. Marculescu. On-chip traffic modeling and synthesis for MPEG-2 video applications. IEEE Trans. on VLSI, 12(1):108-119, 2004.

[16] D. Wiklund, S. Sathe, and D. Liu. Network on chip simulations for benchmarking. In IWSOC, pages 269-274, 2004. 